\title{
Cadenas Cortas de Comercialización y seguridad ALIMENTARIA: EL CASO DE EL MERCADO EL IOO
}

\author{
Laura Martínez Salvador, ${ }^{a}$ Loarry Gabriel Hernández ${ }^{\mathrm{b}}$ \\ y David Alvarado Ramírez ${ }^{c}$
}

Fecha de recepción: 3 de noviembre de 2020. Fecha de aceptación: 27 de abril de 2021 .

$$
\text { https://doi.org/10.22201/iiec.20078951e.2021.206.69732 }
$$

Resumen. En el contexto de la emergencia sanitaria en la Ciudad de México (CDMx), es importante rescatar modelos de abasto alimentario como las Cadenas Cortas de Comercialización (ccC), que pueden materializarse a través de estructuras como El Mercado el 100 (M100). La presente investigación tiene por objetivo analizar el potencial que tiene el M100 para contribuir a la seguridad alimentaria de espacios locales en la CDMX, y definir la forma en cómo estas estructuras respondieron a la emergencia sanitaria actual. Estructuras de ccC como el M100 constituyen modelos de asociatividad agroalimentaria que surgen con redoblada fuerza en momentos de crisis impulsando la seguridad alimentaria local de las urbes.

Palabras clave: seguridad alimentaria; Cadenas Cortas de Comercialización (CCC); abasto alimentario; mercados alimentarios; emergencia sanitaria.

Clasificación JEL: O18; Q12; Q56.

\section{Short Marketing Chains and food security: THE CASE OF El MERCADO EL iOO}

\begin{abstract}
The health emergency in Mexico City (CDMx) has made it vital to revitalize food supply models such as Short Marketing Chains (sMC), which can be constructed via structures such as El Mercado el 100 (M100). This article analyzes M100's potential to contribute to the food security of local spaces in CDMx. Additionally, the article defines how these structures responded to the current health emergency. sMC structures such as the M100 are models of agrifood associativity that emerge with renewed strength in times of crisis, promoting local food security in cities.
\end{abstract}

Key Words: food security; Short Marketing Chains (SMC); food supply; food markets; sanitary emergency.

${ }^{a}$ Universidad Nacional Autónoma de México (UNAM), Instituto de Investigaciones Sociales, México; ${ }^{\mathrm{b}}$ Unam, Posgrado en Ciencias de la Sostenibilidad, México; ${ }^{\mathrm{c}}$ Instituto Politécnico Nacional (IPN), Centro Interdisciplinario de Investigaciones y Estudios sobre Medio Ambiente y Desarrollo (CIIEMAD). Correos electrónicos: laura.martinez@sociales.unam.mx; loarrygabriel@hotmail.com y david.al.rmz@hotmail.com, respectivamente. 


\section{INTRODUCCIÓN}

En la Ciudad de México (CDMx en lo sucesivo), el sistema de abastecimiento alimentario está conformado por redes de flujo y distribución de alimentos situados alrededor de varias estructuras de comercio, tanto para el menudeo como para el mayoreo: supermercados, mercados públicos, tianguis, y "espacios de venta de alimentos orgánicos o agroecológicos" (Pardo y Durand, 2018, p. 473). La existencia de estas últimas estructuras de comercio resulta benéfica para la población, sobre todo ante un panorama de complejidad como la emergencia sanitaria por el virus SARS-CoV-2. La pandemia condujo a autoridades federales y locales de México a aplicar distintas medidas y acciones para la contención de la enfermedad como: esquemas de movilidad limitada, ralentización de actividades productivas, cierre de puntos de venta e intercambio comercial, así como el freno de actividades turísticas y de esparcimiento.

Múltiples actores, organizaciones y otras Estructuras de Abasto Alimentario Alternativas (en adelante EAAA), como el mercado de productores el M100, requirieron reformular sus estrategias de producción, distribución y comercialización de alimentos en la CDMX para contribuir así a la seguridad alimentaria de la urbe. No obstante, factores como las limitadas capacidades productivas de las unidades, la incertidumbre institucional, las dificultades de acceso a materias primas y puntos de venta pueden agudizar las condiciones de vulnerabilidad de estas EAAA y sus actores, así como limitar el acceso a alimentos sanos, acentuando las condiciones preexistentes de inseguridad alimentaria local, y que impacta negativamente a la economía local de las urbes.

En este sentido, la presente investigación tiene por objetivo analizar el potencial que tienen las estructuras de abasto alimentario bajo CCC como El Mercado el 100 (M100), para contribuir a la seguridad alimentaria de espacios locales en la CDMX, así como definir la forma en cómo estas estructuras se ven afectadas bajo contextos adversos como la crisis sanitaria.

Esta investigación se divide en cinco secciones, incluida la introducción, iniciando con una revisión en torno al concepto teórico de la seguridad alimentaria y sus dimensiones, así como de las ccc. En seguida se presenta la metodología utilizada, para dar paso a los resultados y discusiones; en la tercera sección se aprecia el caso de estudio del M100, así como los resultados obtenidos de las encuestas aplicadas. El documento cierra con una sección de reflexiones finales. 


\section{MARCO TEÓRICO}

\section{Seguridad alimentaria. Aproximaciones desde sus dimensiones}

A pesar de existir un consenso global sobre la relevancia que tiene la alimentación para los territorios, en las dos últimas décadas del siglo xxI, los paradigmas en torno a la seguridad alimenaria y los sistemas alimentarios muestran cambios importantes. Por un lado, se rescata el papel social y ambiental que la agricultura tiene, superando la preconcepción de ser sólo una actividad proveedora de alimentos, pasando a considerarse un elemento fundamental en el desarrollo local y regional. Adicionalmente, se revalorizan las relaciones entre los actores del sistema alimentario, dotando de especial importancia a la existente entre productor y consumidor. En este sentido, las EAAA instauran elementos de interés al crear "vínculos entre las áreas rurales y urbanas, a través de movimientos alimentarios alternativos, gestados en oposición a la dinámica globalizadora [y] perfilándose como un vehículo innovador para crear un paradigma alimentario sostenible" (Marsden y Morley, 2014, como se cita en Reina-Usuga et al., 2018, p. 184).

En este sentido, la seguridad alimentaria es un concepto multidimensional (Urquía, 2014) y que en el marco de la Cumbre Mundial de la Alimentación celebrada en 1996 se definió así:

[...] existe seguridad alimentaria cuando todas las personas tienen en todo momento acceso físico y económico a suficientes alimentos inocuos y nutritivos para satisfacer sus necesidades alimenticias y sus preferencias en cuanto a los alimentos a fin de llevar una vida activa y sana (Organización de las Naciones Unidas para la Agricultura y la Alimentación [FAO], 1996, p. 1).

Esta condición presupone la existencia de una serie de dimensiones que son necesarias, más no suficientes, para alcanzar la seguridad alimentaria, las cuales son disponibilidad, acceso, utilización y estabilidad, y que serán, en propuesta, operacionalizadas en los siguientes párrafos:

a) Disponibilidad. El pilar de la disponibilidad implica que los alimentos se encuentren en existencia en los territorios, en cantidades suficientes, para cumplir las presentes y futuras demandas de alimentos. Este pilar "aborda la parte correspondiente a la 'oferta' y se encuentra en función del nivel de producción de alimentos, los niveles de las existencias y el comercio neto" (FAO Y UE, 2010, p. 5). 
Considerando lo anterior, y para el análisis y operacionalización de esta dimensión, se propone realizar un análisis a la disponibilidad, considerando una serie de elementos que pueden llegar a impactar la existencia de los alimentos como:

1) Volumen de producción, bajo el cual se establece la producción y existencia de alimentos;

2) Volumen de ventas de alimentos, con el cual se puede establecer indirectamente un estadio de sostenibilidad económica de las unidades productivas agroalimentarias;

3) Umbral mínimo de capacidades y condiciones que favorezcan la producción, tales como disponibilidad y tenencia de la tierra (ya sean espacios propios, rentados, colectivos-ejidales), disponibilidad de mano de obra, de infraestructura (espacios para almacenar y transformar), maquinaria o equipo de producción y transformación, apoyo financiero público o privado para la producción, transformación o comercialización;

4) Afectaciones y nivel de respuesta ante condiciones medioambientales adversas que puedan impactar negativamente la producción de alimentos, tales como elementos del cambio climático (sequías, inundaciones, plagas o enfermedades, cambios en los periodos de cosecha, cambios en el clima como olas de calor, vientos excesivos, adaptación de ciclos de cultivo).

b) Acceso. Como segunda dimensión de la seguridad alimentaria, implica la consciencia creciente de que la producción, o existencia física de los alimentos, no garantiza que las personas puedan consumirlos, ya que "[los] medios de acceso a los alimentos incluyen el comercio, el trueque, la recolección de alimentos silvestres y las redes de apoyo comunitarias" (FAO y UE, 2010, p. 5). Este elemento es de gran importancia, ya que es necesario reflexionar en torno no sólo a la existencia misma de los alimentos, sino de la posibilidad física y económica de acceder a ellos, asimismo:

[...] debe tenerse presente que el acceso a los alimentos depende de las condiciones del mercado y los precios de los alimentos, así como del poder adquisitivo de las personas, todo lo cual se relaciona a su vez con las oportunidades de empleo y de subsistencia (FAO y UE, 2010, p. 1). 
Considerando lo anterior, desde la perspectiva de los consumidores, los precios son un elemento clave para alcanzar el acceso a los alimentos en el marco de la seguridad alimentaria (Dowler et al., 2011, como se cita en Chiffoleau et al., 2019, p. 183). Tomando en cuenta lo anterior, el acceso puede analizarse a partir de los siguientes elementos, los cuales pueden influir sobre esta dimensión:

1) Nivel de disponibilidad y acceso a medios de distribución y espacios de venta. En esto se considera la posibilidad para actores de la cadena de valor agroalimentaria de acceder a espacios físicos, ya sean públicos o privados, para el intercambio de alimentos.

2) Condiciones de infraestructura de caminos, carreteras, vías y calles para el abasto y transporte de productos a puntos de venta, este elemento abona al tema de la movilidad, lo que es una condición necesaria para garantizar el acceso físico a los alimentos.

3) Uso de otras estrategias para garantizar el abasto alimentario (comercialización y venta, como comercio electrónico, uso de plataformas de difusión y canastas alimentarias).

4) Nivel de precios ofrecidos al público, en comparación con aquellos alimentos símiles ofrecidos en puntos de intercambio, como son centrales de abastos u otros mercados.

5) Elementos que pueden impactar el nivel de precios producto de una serie de condiciones como la presencia de intermediarios, aumentos en los costos de producción e incrementos en los costos de comercialización.

c) Utilización. Por lo que hace a la utilización se entiende "como la forma en la que el cuerpo aprovecha los diversos nutrientes presentes en los alimentos" (FAO y UE, 2010, p. 6). Esta es una concepción multidimensional, pues involucra aspectos que van más allá de los alimentos; tiene que ver con la higiene, el acceso al saneamiento, al agua, a la información sobre la sanidad de los alimentos y se ve reflejada en cómo los mismos son aprovechados por los individuos (en cantidad, en suficiencia calórica, en proporción y acceso a nutrientes básicos). Considerando la conceptualización de la dimensión utilización, el análisis de ésta puede realizarse, y ser operacionalizada como en los casos anteriores, al reflexionar en torno a aquellos elementos que pueden influir tanto en la calidad e inocuidad de los alimentos, como en el conocimiento que el consumidor tenga del alimento en relación con 
el contenido nutricional, el grado de inocuidad y los estándares de calidad bajo los cuales la producción y transformación de alimentos se desarrolló. Estos elementos son:

1) Elementos de identificación con los que cuenta el producto final, para el caso de alimentos transformados: información nutrimental, ingredientes contenidos, datos del productor, empacador, transformador o importador. Estos elementos son de importancia ante la necesidad que tiene el consumidor de conocer el contenido nutricional de los alimentos, así como las medidas de higiene e inocuidad implementadas durante el proceso productivo. Adicionalmente, como parte de los elementos de la utilización de los alimentos, es pertinente incluir otros sellos de calidad y certificaciones en los productos alimenticios, toda vez que constituyen un reconocimiento institucional de estándares de calidad o procesamientos especializados, como es el caso de los productos orgánicos o agroecológicos. En este apartado se incluyen otro tipo de reconocimientos, como el comercio justo, buenas prácticas ambientales, marcas colectivas o sellos con reconocimiento territorial o denominación de origen.

2) Otros distintivos de calidad, o sellos de origen como certificados de calidad (e.g. Norma Oficial Mexicana), certificado de producto orgánico, certificado de producto agroecológico, certificado de comercio justo, certificado de buenas prácticas ambientales.

d) Estabilidad. Considerando que las primeras tres dimensiones son pasos necesarios en la construcción de la seguridad alimentaria, la estabilidad es una dimensión transversal que se alcanza cuando las primeras tres dimensiones también tienen permanencia en el largo plazo, de forma tal que las variaciones, producto de factores endógenos o exógenos al territorio, no comprometen la provisión de alimentos sanos a la población de una región (FAO Y UE, 2010).

Desde una perspectiva macro, esta estabilidad puede implicar la existencia de políticas públicas diseñadas para fortalecer la producción, equilibrar el acceso a los alimentos e impulsar la disponibilidad y adquisición de alimentos inocuos. Sin embargo, se tienen elementos que impactan sobre las pequeñas unidades agroalimentarias, productores primarios o sobre las agroindustrias. En este sentido, el elemento de estabilidad puede analizarse a partir de: 
1) Existencia de apoyos y estímulos diseñados para la continuidad de la actividad agroalimentaria. En este punto se definen acciones o estrategias que instituciones, públicas o privadas, deben realizar a fin de fortalecer la resiliencia de las cadenas agroalimentarias como créditos a la producción, estímulos directos o indirectos a la producción, construcción de redes de suministro y abasto confiables, campañas de difusión y promoción, apoyo en la mejora de infraestructura, apoyo para aumentar la seguridad y sanidad de los espacios de venta, asesoría técnica y acompañamiento para mejorar la producción o transformación.

2) Elementos socioculturales y construcción de capital social que implica la valorización de tradiciones, cultura, usos y costumbres propios del quehacer alimentario; así como por la conformación de redes de colaboración y confianza entre actores e incluso la presencia de procesos de relevo generacional.

3) Estabilidad institucional, certidumbre con la que los actores toman decisiones de inversión o compra, así como para proyectar ventas y favorecer la toma de decisiones productivas. Por otra parte, la estabilidad institucional se ve reflejada en la necesidad de continuidad administrativa e incluso en la existencia de políticas, estrategias productivas y sociales (Urquía, 2014) para el desarrollo de actividades agroalimentarias bajo estructuras de abasto alternativas.

Sin embargo, para que existan niveles de seguridad alimentaria, es necesario integrar las dimensiones mencionadas con el fin de generar sistemas de abasto alimentario sostenibles y funcionales a través de estructuras alternativas conformadas "por la producción, el acopio, el almacenamiento, la transformación, el consumo [y] la comercialización de alimentos" (Bassols et al., 1994, p. 36). Asimismo, es importante considerar que el anterior constructo de seguridad alimentaria mantiene una escala analítica amplia, de nivel macro y de escalas nacionales, por lo que es importante matizar que, si bien este constructo puede proporcionar elementos teóricos adecuados para el análisis de cada uno de los pilares que la componen, es conveniente tomar en cuenta que, para efectos de este trabajo de investigación, se entienden y reconocen los alcances de incidencia que las EAAA como el M100, erigido como materialización de las CCC, a los pilares mencionados pueden ser propios de una escala micro. Lo anterior no debe considerarse una debilidad, sino más bien una ventana de oportunidad para impulsar y fortalecer estas estructuras alternativas, visibilizando la contribución e importancia de estos mercados a la seguridad alimentaria local en espacios urbanos. 


\section{Cadenas Cortas de Comercialización para la seguridad alimentaria}

Las CCC se definen como las "formas de comercio basadas en la venta directa de productos frescos o de temporada [con baja o nula intermediación] entre productores y consumidores" (FAO, 2016, p. 1), favoreciendo la cercanía geográfica, impulsando la agricultura familiar, reduciendo los eslabones de la cadena de valor alimentaria y generando vínculos de confianza (Padilla y Oddone, 2016). Estas CCC "son concentraciones espaciales de explotaciones agrarias, empresas e instituciones en red, especializadas territorialmente en torno a un subsector o subsectores agroalimentarios determinados" (Delgadillo y Sanz, 2018, p. 10), cuyas características principales están en función de la cercanía entre los espacios de producción, distribución, comercialización y consumo, así como por la "gran participación de pequeños agentes [que comprenden unidades de] economía familiar [y] artesanal y el flujo orientado hacia mercados locales y regionales" (Azevedo, 2009, p. 11).

Para Goodman et al. (2012), la percepción de un modelo de alimentación industrial insostenible, propio y característico de un modelo de abasto alimentario globalizador, pudo generar la emergencia de movimientos y espacios de producción y consumo de alimentos basados en principios éticos y responsables alrededor de lo orgánico, y cuyas estructuras, como las CCC "[re]presentan una promesa de diferenciación, [trascendiendo] los modelos de cadenas largas de suministro, que representan la construcción social de nuevos modelos económicos" (Chiffoleau et al., 2019, p. 182), los cuales se contraponen a tendencias hegemónicas homogeneizadoras del consumo, y que caracterizaron procesos comerciales propios de la globalización, como la introducción de "empresas transnacionales agroalimentarias [dando paso a] cambios en el consumo de alimentos con base en la expansión de alimentos preparados e industrializados" (Pensado, 2003, p. 204), así como cambios en el patrón alimenticio, un aumento en la importación de alimentos de dudoso aporte nutricional, la integración de cadenas de comida rápida (fast food) y el aumento de cadenas de autoservicio.

Ante los cambios y transformaciones de las dinámicas de producción, transformación, distribución y consumo de alimentos de este periodo, surgieron alternativas de abasto alimentario que pueden considerarse así en la medida que representan puntos de intercambio de bienes agroalimentarios que pueden clasificarse como artesanales, tradicionales, agroecológicos u orgánicos, y cuyas dinámicas de comercialización promueven CCC, comercio justo y la distribución de beneficios entre los actores sociales. Estas estructuras se 
convierten en potenciadoras del desarrollo territorial, cuestionando el modelo tradicional agroindustrial dominante a través de sus diversas modalidades (Renting et al., 2003), siendo de especial interés el papel de las CCC.

Ante lo polisémico del concepto de CCC, es importante mencionar dos enfoques bajo los cuales se realiza el abordaje analítico de estas estructuras. Desde el enfoque de la oferta, las CCC están basadas en la interacción cara a cara entre productor y consumidor (Korhonen et al., 2017) y pueden estar conformadas por sistemas de autoabastecimiento, como los huertos colectivos y algunos tipos de agricultura urbana, jardines comunitarios o jardines individuales; cadenas de intercambio comercial, mercados de productores, tianguis orgánicos o agroecológicos, mercados de trueque, ventas en carretera y agricultura comunitaria. Por otra parte, desde el lado de la demanda o el consumo, las CCC rescatan con particular importancia el origen, la calidad del alimento, la transparencia, la trazabilidad, el acceso y el precio de los alimentos, atributos que impactan directamente en el consumo.

\section{METOdología}

Para la presente investigación se realizó una revisión del constructo de seguridad alimentaria y se operacionalizaron dimensiones de la misma a partir de un estudio de caso (Yin, 2003). Para profundizar en torno al estudio de caso, se utilizó un sondeo no probabilístico a partir del muestreo en bola de nieve, estableciendo como canal de comunicación a la representante legal del M100. Para llevar a cabo este sondeo se diseñó un cuestionario semi-abierto, en formato electrónico, que fue aplicado a productores, transformadores y comercializadores pertenecientes al M100 durante el mes de julio de 2020.

Actualmente, el M100 se conforma por 46 productores pertenecientes a distintos ramos (alimentos preparados, frutas, verduras y hortalizas, productos de higiene personal, artesanías, alfarería y productos de limpieza). Sin embargo, dado el interés de esta investigación en abonar al conocimiento en torno a las prácticas agroalimentarias, se consideró importante incluir sólo a productores relacionados al ramo alimenticio. En un primer momento, se solicitó apoyo a la representante legal para que, a través de su contacto inicial, hubiese respuesta al cuestionario por parte de los productores; al término de la investigación (octubre 2020), el cuestionario fue contestado por una decena productores del M100.

El cuestionario se conformó por 49 reactivos dividido en seis grandes secciones, dentro de las cuales se incluyeron cuestionamientos para conocer ge- 
neralidades de cada productor, así como de su actividad. Asimismo, los cuatro pilares de la seguridad alimentaria se operacionalizaron a partir de los elementos mencionados en el apartado teórico entorno a la disponibilidad, el acceso, la utilización y la estabilidad, desde las condiciones o estímulos necesarios para la continuidad de la actividad agroalimentaria. De igual forma, se buscó conocer condiciones, acciones y estrategias de producción y comercialización de los encuestados previo a la crisis sanitaria por la pandemia por Covid-19, así como durante ésta (hasta el cierre de esta investigación continuaba activa).

La información obtenida de los cuestionarios sirvió para conformar una base de datos que fue sistematizada temáticamente y, posteriormente, analizada a través de hojas de cálculo de Microsoft Excel para el tratamiento de información cuantitativa. Las respuestas fueron revisadas e integradas al análisis a partir de seleccionar puntos de convergencia resaltando las diferencias entre cada productor.

\section{RESULTADOS Y DISCUSIÓN}

\section{El Mercado El 100 en la Ciudad de México}

El M100 es una Asociación Civil que se constituyó como una alternativa de comercialización constante $y$, sin intermediarios, entre productores $y$ consumidores, siendo el primero en su tipo en tener el reconocimiento de Certificación Orgánica Participativa que otorga la Secretaría de Agricultura y Desarrollo Rural (SADER) y el Servicio Nacional de Sanidad, Inocuidad y Calidad Agroalimentaria (senAsica) (Time Out México, 2020; M100, 2020). El M100 lo conforman 46 productores, de los cuales $89 \%$ se dedican al giro alimenticio, y el 11\% restante a diversas actividades como alfarería y elaboración de artesanías, cuidado personal, y a la comercialización de productos de limpieza biodegradables (M100, 2020).

Este mercado comenzó a operar en 2009 (López y Agencia El Universal, 2011), y su nombre surge de la respuesta a la idea de que los productores deben provenir de regiones no más allá de 100 millas (160 kilómetros) a la redonda. No obstante, aunque el mercado se constituyó con un enfoque de producción local materializado en la cercanía de la producción a la CDMx, las dinámicas de los consumidores provocaron que los criterios de distancia se flexibilizaran (Salgado-Sánchez y Castro-Ramírez, 2016). En la actualidad, 50\% de los productores provienen de la CDMx, 32\% del Estado de México, 7\% de Morelos, $2 \%$ de Puebla (mismo porcentaje para las entidades de Veracruz y Querétaro), y 5\% de Tlaxcala (M100, 2020). 
Es importante destacar que el M100 posee una serie de características organizacionales basadas en la colaboración interna, la inclusión de actores y el fortalecimiento de los vínculos de confianza, entre productor y consumidor y productor-productor, de igual forma se caracteriza por ser un modelo de asociación comercial con ausencia de intermediarios, por lo que constituye un sólido ejemplo de la materialización de las CCC. Asimismo, esta forma de abasto alimentario genera una relación sostenible entre el campo y los puntos de consumo urbanos como la CDMx, logrando un espacio donde se fomenta la agricultura familiar, diversas formas de trabajo y el comercio justo a través de la producción y el consumo responsable.

\section{El Mercado El 100 y la seguridad alimentaria en la CDMX en el marco de la crisis sanitaria}

Las preguntas implementadas en el cuestionario electrónico fueron diseñadas a fin de obtener información que permitiera conocer los aportes que esta estructura de abasto alimentario tiene sobre la seguridad alimentaria de espacios locales en la CDMX, desde los diferentes pilares previamente descritos y para conocer, entre otros aspectos, retos y oportunidades de la comercialización de sus productos alimenticios antes, durante y posterior a la emergencia sanitaria de Covid-19 en la CDMx. A continuación se muestran los resultados de la encuesta aplicada a una decena de productores.

Es de interés rescatar que, de los productores que respondieron al cuestionario, $40 \%$ son mujeres y $60 \%$ hombres; asimismo, considerando el interés de abonar al conocimiento en torno al ramo alimenticio, 100\% de los encuestados son productores de bienes agroalimentarios o productores de utensilios para la actividad alimentaria. En este sentido, es importante destacar el carácter heterogéneo de los productores y sus actividades (productos derivados de nopal y de otros cereales y granos; plantas comestibles; frutas, verduras y hortalizas; vainilla y otras vainas; huevos y carne orgánica; café y derivados; cacao y sus derivados; y utensilios del ramo alimenticio), esta variedad de productores contribuye a validar la información de la población del M100, ya que si bien los demás productores del ramo alimenticio no encuestados guardan diferencias con los analizados en este trabajo, podría considerarse que forman parte de algunas de estas categorías productivas mencionadas. De lo anterior se desprende el hecho de que $50 \%$ de los encuestados participan activamente en todos los pilares de la cadena de valor agroalimentaria, siendo sólo $20 \%$ en la producción primaria, $10 \%$ realiza sólo actividades de transformación, 
y otro $20 \%$ realiza actividad de transformación y comercialización. Lo anterior presenta un productor con conocimientos integrales de los eslabones de la cadena agroalimentaria, lo que podría contribuir a formular estrategias de desarrollo productivo más completas.

Las unidades agroindustriales o productivas a las que pertenecen los encuestados son en un $80 \%$ de base familiar; iniciaron sus actividades, en $40 \%$ de los casos, en los últimos cinco ańos, y un 60\% inició actividades en el M100 también durante ese periodo.

El ingreso al M100 representó para estos productores el aumento de sus ingresos económicos, producto de mayores ventas; un incremento en la capacidad productiva y la posibilidad de obtener certificados de producción agroecológica u orgánica, así como el acceso a cursos y capacitaciones productivas. Todo lo anterior a la par de la generación de empleos.

\section{La dimensión disponibilidad en el M100 para la seguridad alimentaria}

Como se menciona y propone en el punto teórico y metodológico, la disponibilidad, como dimensión de la seguridad alimentaria, puede ser operacionalizada a partir de una serie de elementos que tienen impacto y efecto sobre el nivel de producción de los alimentos.

En este sentido, los resultados de la encuesta arrojaron que el volumen de producción de las unidades es limitado, tendencia que se ha mantenido en los últimos años, con una ligera tasa de ascenso. No obstante, dificulta la inserción de los productores en cadenas de comercialización como supermercados o tiendas de autoservicio para el escalamiento productivo.

La disponibilidad de alimentos en este mercado puede verse afectada por el volumen de producción, la tenencia de la tierra y la adquisición o uso de espacios de trabajo, ya sean propios, rentados o colectivos. En este tema es interesante mencionar que para $60 \%$ de los productores encuestados los espacios de trabajo son de uso propio, mientras que $40 \%$ de los espacios son rentados, lo que puede representar un elemento de vulnerabilidad para los que no poseen lugares de trabajo propios.

Por otra parte, el nivel de producción puede verse afectado por otros elementos generados por afectaciones directamente relacionadas con el cambio climático como sequías, inundaciones; plagas o enfermedades; cambios la duración o intensidad de las estaciones (como olas de calor o vientos excesivos), por lo que se cuestionó respecto a estos elementos y fue posible rescatar que $60 \%$ de los encuestados vio afectados su nivel de producción debido a las se- 
quias o a la duración del periodo de estiaje, mientras que $40 \%$ ha presentado afectaciones por inundaciones; $50 \%$ asegura haber tenido problemas en el nivel de producción debido a las plagas, y $60 \%$ de los productores reportó impactos en la producción relacionados con las olas de calor.

En el último año el volumen de ventas como indicador de una posible sostenibilidad económica para los productores tuvo una tendencia a la baja, condición que se recrudeció a partir de las restricciones de movilidad instauradas en la CDMX, y por un efecto de contracción de la demanda interna alimentaria a partir de la emergencia sanitaria, por lo que el volumen de ventas disminuyó hasta en un 70\%. Es importante tener en cuenta esta tendencia, toda vez que la actividad del M100 representa para $80 \%$ de los productores su principal ingreso.

En este mismo sentido, en torno a las afectaciones por la emergencia sanitaria sobre elementos de la producción (véase figura 1), es interesante mencionar que la percepción en torno a la disponibilidad y acceso a materias primas (semilla, abonos, fertilizantes o sistemas de riego) ha cambiado hacia una percepción más negativa, pasando de ser considerada como "Buena" o "Muy Buena" para 70\% de los consultados (7 de 10 productores) a 30\% (3); de "Regular" 30\% (3) a 50\% (5), y a considerarse "Mala" en 20\% (2).

Figura 1. Percepción en el nivel de disponibilidad y acceso a materia prima previo y durante Covid-19, 2020

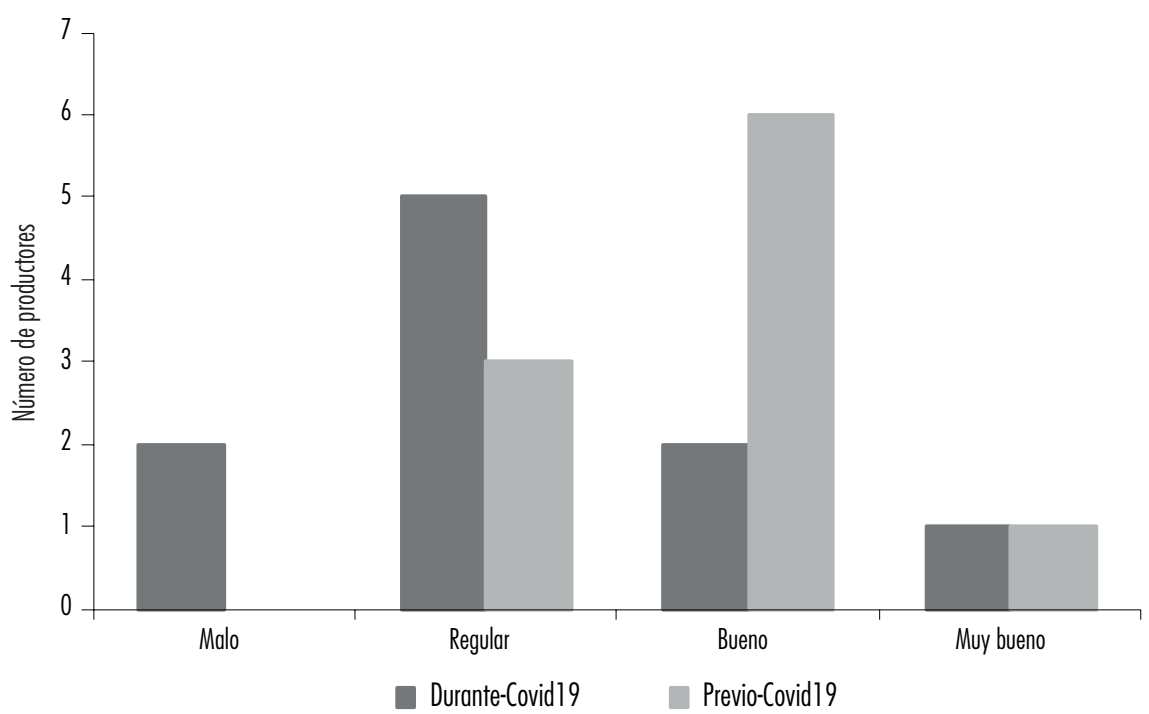

Fuente: elaboración propia con base en las encuestas. 
Por otra parte, en cuanto a disponibilidad y acceso de mano de obra (véase figura 2), se percibió una disminución en disponibilidad de "Buena" o "Muy Buena" de 60 a 30\% (6 a 3), y de "Muy Mala" a "Mala" de 0 a 20\% (0 a 2); lo anterior debido a las restricciones de movilidad para el personal requerido en el proceso de sembrado, cultivo, recolección y transformación de alimentos.

Desde otro punto, en cuanto al acceso a espacios para producir, almacenar y transformar, la percepción pasó de "Bueno" o "Muy Bueno" en 70 a 30\% (7 a 3), mientras que pasó de una percepción de "Mala" de 0 a 30\% (0 a 3) (véase figura 3).

Todo lo anterior generó una disminución en el volumen de producción para 60\% (6) de los actores, por lo que se infiere que si bien estas unidades de abasto alimentario poseen el potencial de abonar a la dimensión de la disponibilidad de alimentos como pilar de la seguridad alimentaria, ante situaciones exógenas adversas, como la crisis de emergencia sanitaria, sus condiciones y capacidad de producción de alimentos se vulneran ampliamente, pudiendo tener afectaciones sobre uno de los eslabones del abasto alimentario en los centros urbanos.

Figura 2. Percepción en el nivel de disponibilidad y acceso a mano de obra previo y durante Covid-19, 2020

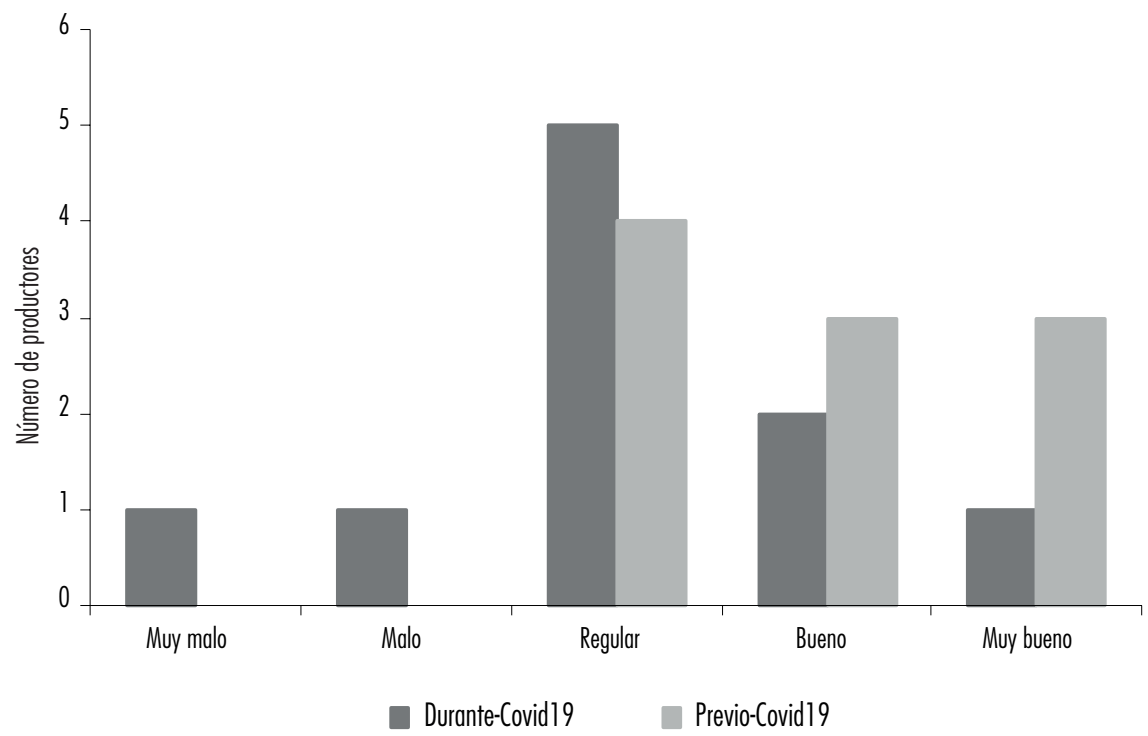

Fuente: elaboración propia con base en las encuestas. 
Figura 3. Percepción en el nivel de disponibilidad y acceso a infraestructura previo y durante Covid-19, 2020

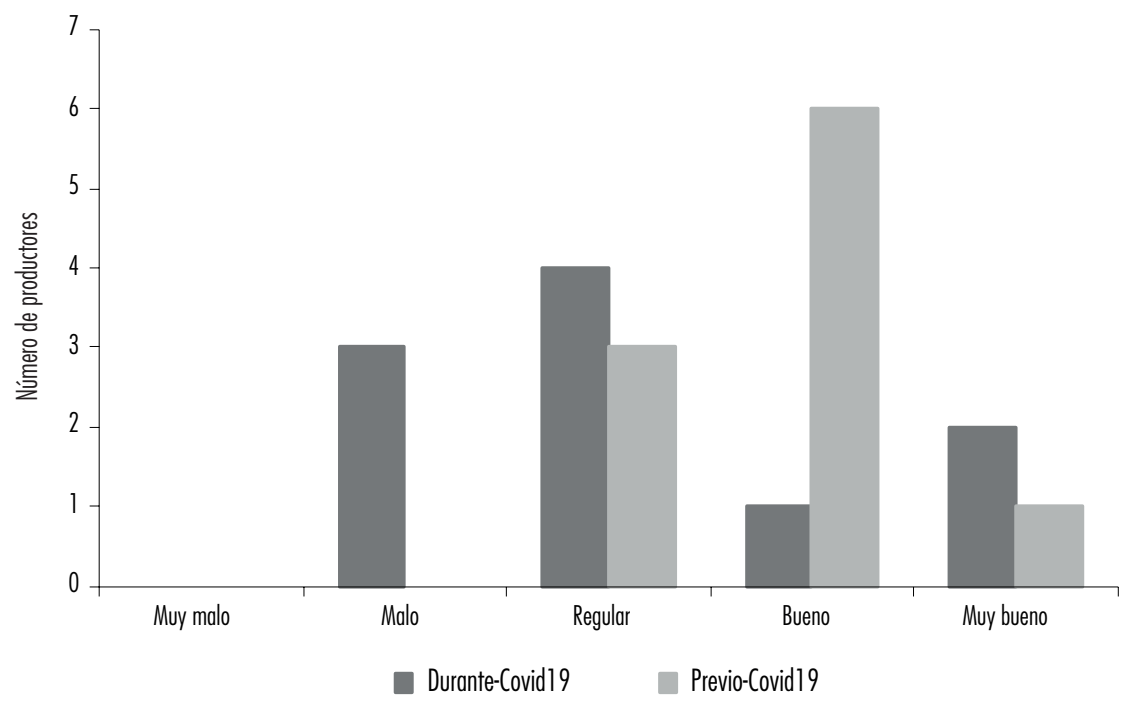

Fuente: elaboración propia con base en las encuestas.

\section{La dimensión acceso en el M100 para la seguridad alimentaria}

El acceso a los alimentos en el M100 puede verse afectado por una serie de condiciones, como el acceso a medios de distribución, infraestructura de caminos para el abasto y transporte de productos, así como estrategias, tradicionales y alternativas, para la comercialización de alimentos o el nivel de precios. En este sentido, y siguiendo con la propuesta metodológica en torno a identificar y operacionalizar el acceso a los alimentos, los resultados de las encuestas arrojaron que $40 \%$ (4) de los participantes coincidieron en que el estado actual de la infraestructura de caminos, carreteras, vías y calles para el abasto y transporte de productos es "Muy Bueno". Sin embargo, 20\% (2) considera que estos accesos no son suficientes, debido a que, al tratarse de una entidad densamente poblada, la CDMX es un punto de intercambio comercial con movilidad limitada y dificultad de tránsito. Por otra parte, a partir de la emergencia sanitaria, la percepción sobre la disponibilidad y acceso a medios de distribución y espacios de venta pasó de "Regular" en 80\% (debido a que la instauración de mercados de este tipo en espacios públicos ha constituido desde siempre un elemento de dificultad administrativa) a "Muy Malo" o 
"Malo" en 60\% (8 a 6), producto de las restricciones de movilidad, el cierre de comercios y las medidas de distanciamiento social.

En cuanto a la movilidad para el suministro o venta durante el periodo de contingencia sanitaria, 30\% de los encuestados la considero como "Buena" o "Muy Buena" debido a la disminución en el tránsito vehicular. Sin embargo, $60 \%$ (6) la consideró "Mala" o "Regular" debido a que fue posible percibir restricciones en el transporte público, lo que constituye una desventaja para aquellos productores que no cuentan vehículo propio para las actividades de suministro y comercialización.

Uno de los principales retos, percibido por parte de los participantes, fue el manejo de los costos de producción y su repercusión en los precios de venta, elemento que, junto con la capacidad del poder adquisitivo de la población, constituye un punto de relevancia cuando al acceso de alimentos se refiere.

En este sentido, es importante mencionar que la ausencia de intermediarios, con base en lo mencionado en el ejercicio de la encuesta, ha permitido al $70 \%$ (7) de los productores ofrecer mejores precios. No obstante, debido a la pandemia, este mismo porcentaje de entrevistados tuvo que modificar (al alza) sus precios de venta, lo que podría significar que los bienes alimentarios que ofrecen sean considerados como "bienes de lujo".

Lo anterior puede constatarse, a su vez, a través del siguiente análisis comparado de precios ofrecidos al público por parte del M100 y de sus símiles ofrecidos por otros puntos de intercambio, como la Central de Abastos de la CDMX. La búsqueda de precios comparativos permitió identificar que en productos considerados como básicos y de mayor consumo en la ciudad (Secretaría de Desarrollo Social [SEDESOL], 2016), existe un incremento que va desde 50 hasta 200\%. Ante esto, los actores de EAAA, bajo modelos de CCC como el M100, requieren generar estrategias para hacer frente a dos cuestiones: por un lado, se requieren planes que aborden la disminución de los costos productivos y la búsqueda de economías de escala, condición que muestra ya un avance, si se considera que son estructuras libres de intermediarios. Por otro lado, es necesario realizar un mayor esfuerzo de concientización para impulsar el consumo de bienes alimentarios, como los encontrados en este mercado, que se consideran como inocuos, sanos, de producción local, y que a la vez contribuyen a mejorar las condiciones de vida de familias agricultoras. Esto requiere de estrategias integrales vinculantes que en ocasiones pueden sobrepasar las capacidades de los actores primarios.

A raíz de la emergencia sanitaria, el M100 comenzó un proceso de transición al comercio electrónico, tanto desde la plataforma común del M100, como de forma individual. 
En este sentido, los resultados de este ejercicio permiten apreciar que otro de los principales retos enfrentados por los productores del M100 es la necesidad de realizar cambios logísticos y productivos para atender a los nuevos protocolos de higiene y sanidad requeridos. Asimismo, ha sido necesaria una constante y completa transformación del acercamiento a los consumidores y del abasto alimentario, ya que ante la limitada movilidad, 100\% de los entrevistados estableció que ha tenido que hacer uso de otras estrategias de comercialización y venta, dentro de las que destacan el uso de las Tecnologías de la Información y la Comunicación través de plataformas de comercio electrónico, creación de páginas web, uso de redes sociales, mensajería electrónica y entregas a domicilio.

En este punto del acceso a la alimentación, $50 \%$ de los consultados considera que podrían mejorarse las condiciones de comercialización si se fortalecieran los puntos de intercambio, incrementando las medidas sanitarias y garantizando la seguridad de los consumidores, quienes, ante el cambio de normativas sanitarias en la CDMX, han comenzado a asistir nuevamente al M100.

\section{La dimensión utilización en el M100 para la seguridad alimentaria}

Considerando que la utilización, como pilar de la seguridad alimentaria, implica no sólo la inocuidad, calidad nutricional y sanidad de los alimentos, sino que también puede indicar que estos elementos sean bien conocidos por el consumidor, la utilización es multidimensional y considera que los productos cuenten con datos de identificación para venta y consumo. En el caso de los productos ofrecidos en el M100, se encuentran presentes, por lo que hace a la marca o nombre de identificación, en $80 \%$ de la producción de los consultados, $50 \%$ cuenta con información nutrimental, y $70 \%$ tiene datos de contacto del productor o empacador.

En cuanto a otros sellos o distintivos de calidad, es interesante mencionar que $30 \%$ de los productores cuenta con certificaciones oficiales que garantizan estándares de calidad productiva como las Normas Oficiales Mexicanas (NOM), mientras que $40 \%$ ha iniciado procesos para su obtención. Por otro lado, las certificaciones orgánicas o agroecológicas han sido obtenidas por $40 \%$ (4) de los entrevistados. No obstante, los reconocimientos de comercio justo son un elemento ausente en 90\% (9) de los productores. Lo anterior puede deberse, en parte, al poco conocimiento que se tiene en materia de certificaciones o reconocimiento de este tipo.

La información respecto al lugar de origen está presente y visible en los productos del $50 \%$ de los entrevistados, mientras que la denominación de 
origen (protección institucional) es un elemento presente solamente en los productos del $20 \%$ (2) de los entrevistados.

Ante estos resultados, es importante cuestionarse respecto a la funcionalidad de estas figuras de protección institucional. En ocasiones, el interés para obtener dichos reconocimientos puede estar en función de los siguientes elementos: la solicitud por parte de la demanda, la cual impulsa a la oferta a certificarse; la capacidad, técnica o productiva de las unidades para cumplir requisitos y estándares de calidad solicitados por las certificaciones y, por último, pero no por ello menos relevante, el costo de las certificaciones y la capacidad financiera de los actores para acceder a ellas.

\section{La dimensión estabilidad en el M100 para la seguridad alimentaria}

Finalmente, la dimensión de la estabilidad en la seguridad alimentaria se enmarca en la propia definición, al establecer que las anteriores condiciones (disponibilidad, acceso y utilización) deben permanecer en todo momento y para todas las personas. Por lo tanto, se convierte en un elemento transversal que puede ser influido por múltiples factores. Estos factores, en el caso de estructuras de abasto bajo enfoques de Ccc, se refieren a la existencia de apoyos y estímulos necesarios para la continuidad de la actividad agroalimentaria, la presencia o ausencia de elementos socioculturales o tradicionales que pueden tener efectos sobre la actividad y el quehacer agroalimentario, la construcción de redes de confianza entre actores, lo que es propiamente una característica de los Ccc, así como un ambiente institucional estable y que ofrezca certeza para el desarrollo de sus actividades.

Estos elementos fueron utilizados en este documento como indicadores indirectos de esta condición de estabilidad y operacionalizados en el cuestionario. Los resultados mostraron que $80 \%$ (8) de los encuestados asegura no contar con ninguna fuente de apoyo, mientras que sólo 20\% (2) mencionó hacer uso de apoyos directos provenientes de instituciones gubernamentales locales.

Por otra parte, $80 \%$ (8) de los participantes consideran que la actividad que realizan, en el marco del M100 y como estructura de CCC, aumenta la confianza en otros colaboradores, pero también con los consumidores, con quienes han podido crear vínculos de lealtad, basados en el intercambio de conocimiento y experiencias en torno a la producción de alimentos y a su transformación, lo que constituye una de las mayores fortalezas del M100.

Otro elemento relevante en la búsqueda de la estabilidad y continuidad de la actividad agroalimentaria lo constituye el relevo generacional. En este senti- 
do, es importante mencionar que, de los entrevistados, $40 \%$ (4) considera que las generaciones de jóvenes, quienes usualmente son hijos o familiares directos de los productores, no muestran interés por continuar con las tradiciones alimenticias, ni por reproducir o conservar el saber-hacer alimentario, lo que podría poner en riesgo la continuidad de la actividad, así como conducir a la pérdida del patrimonio cultural y tradicional.

Adicionalmente, 40\% (4) considera que, ante el panorama adverso provocado por la pandemia, no es posible contar con certeza económica o financiera que permita realizar compras o inversiones para la producción, situación que se repite para la proyección de ventas. En este mismo tenor, $60 \%$ de los consultados consideró que los cambios de la localidad, en este caso la alcaldía Cuauhtémoc donde se encuentra permanentemente ubicado el M100, tienen impactos negativos en la comercialización de alimentos. Lo anterior puede explicarse debido a que los cambios políticos, en general, vienen aparejados de cambios administrativos, lo que ocasiona que las gestiones logísticas y operativas para la funcionalidad del M100, y el aprovechamiento del espacio público, se vean trastocadas al ser necesario establecer negociaciones con nuevos actores y nuevas administraciones locales.

En este mismo sentido, y en cuando a la estabilidad, es importante mencionar que, en una revisión realizada a una serie de monitores agropecuarios desarrollados por instituciones internacionales, como el Instituto Interamericano de Cooperación para la Agricultura (IICA), no ha sido posible identificar una estrategia, local o federal, que esté enfocada en impulsar o proteger estas formas de abasto alimentario, o a las CCC, e incluso para para la salvaguarda de bienes con características propias de la agricultura agroecológica, orgánicas o de pequeña producción familiar. Esto a la par de la ausencia de continuidad institucional, puede constituir una debilidad sistémica bajo las cuales palidecen las estrategias de fortalecimiento a pequeños grupos de productores para la seguridad alimentaria (Instituto Interamericano de Cooperación para la Agricultura [IICA], 2020).

\section{REFLEXIONES FINALES}

Es importante tomar en consideración que si bien, aunque las EAAA, bajo modelos de CCC, no constituyen una solución integral al problema de la seguridad alimentaria de espacios locales en la CDMX, sí pueden considerarse un avance hacia la instauración de canales de comercialización que favorecen e impulsan el desarrollo territorial, tales como la construcción de vínculos de 
confianza entre productor y consumidor; instauración de procesos productivos agroecológicos y sostenibles, conservación del valor agregado en los territorios productores; generación de derramas positivas tanto en los puntos de intercambio; e incluso preservación de tradiciones culinarias y el saber-hacer alimentario.

Sin embargo, no debe dejarse de lado que estructuras como el M100, que impulsan la CCC, así como otras formas de producción alimenticia de pequeńa escala como la agricultura familiar (la cual también adolece de limitadas capacidades tecnologías y productivas), si bien se erigen como alternativas de abasto alimentario impulsando la seguridad alimentaria de los espacios locales de las urbes como la CDMX, tienen desde su origen características que pueden limitar en mayor o menor medida a las economías de escala, y se enfrentan continuamente al reto de combinar la tradicional artesanal de la producción con la estandarización de los procesos, e incluso con la obtención de certificaciones de calidad, higiene e inocuidad; elementos, que permitirían incrementar la confianza del consumidor, e incluso favorecer la inserción de estos bienes a mercados de mayor valor agregado. Estas limitadas capacidades tecnológicas, productivas e incluso organizacionales, de cada pequeña unidad productiva, se reproducen en ocasiones, ya que se observan pocos incentivos al cambio al insertarse a estructuras como el M100, el cual ha logrado instaurar un modelo funcional que puede ser replicable pero que puede tener dificultades al escalamiento. Estas condiciones se muestran como ventanas de oportunidad, cuya debilidad se agudiza cuando situaciones adversas como la pandemia se suscitan. Aunque es importante destacar que no es el caso de todas las unidades encuestadas ni de todas las del M100.

Considerando lo anterior, es importante mencionar que, analizar el potencial que tienen las CCC, ejemplificadas con el caso del M100 y su contribución a las cuatro dimensiones de la seguridad alimentaria en espacios locales como la CDMX, permite establecer que este mercado requiere de la atención de los elementos previamente mencionados, por lo que se convierte en una ventana de oportunidad al poner a disposición de los consumidores locales alimentos sanos, frescos, inocuos y de temporada. En este sentido, y abonando a la disponibilidad, los canales de abastecimiento, puntos de venta y medios de distribución y comercialización de alimentos, aunque perfectibles, muestran un potencial de crecimiento, aunque puede quedar en ocasiones desaprovechado debido a las características propias de las estructuras organizativas mencionadas previamente, lo que puede restringir también el acceso continuo de los alimentos (estabilidad). De igual forma, y en el sentido del acceso, en la medida en que los niveles de producción puedan verse incrementados solven- 
tando las anteriores limitantes capacidades mencionadas, los costos de producción podrían disminuir, favoreciendo mejores precios al consumidor lo que, aparejado a la disminución o eliminación absoluta de intermediarios propios de una estructura de CCC, puede generar un círculo virtuoso de rendimientos suficientes para los productores y precios accesibles para los consumidores. Por otra parte, dado que estas EAAA bajo modelos de CCC favorecen e impulsan la producción bajo estándares de calidad e inocuidad, y sistemas orgánicos agroecológicos. Estas estrategias de CCC favorecen la dimensión utilización de la seguridad alimentaria de espacios locales en la CDMx, lo que se ve incrementado por la búsqueda constante que cada uno de los integrantes de estos mercados lleva a cabo para acceder a sellos de calidad y certificaciones que aumentan la confianza del consumidor en los bienes adquiridos. Lo anterior de la mano de un continuo y estable proceso de vinculación entre consumidor y productor.

Igualmente importante son los modelos de asociatividad agroalimentaria, que surgen con redoblada fuerza, mostrándonos que incluso en la adversidad, el engranaje de abasto alimentario no se detiene, es resiliente, muestra adaptabilidad y flexibilidad que, aunque a pequeña escala, refleja el espíritu perseverante de productoras y productores, quienes logran generar vínculos fuertes entre los espacios productivos rurales y los centros de consumo urbano. En este sentido, es de gran relevancia el rescate de buenas prácticas de EAAA, bajo modelos de ccC como el M100, fundamentado en el fortaleciendo de los vínculos de confianza entre productor y consumidor, lo que también permite conocer estos modelos de asociatividad, abiertos e inclusivos, ante circunstancias adversas.

La seguridad alimentaria es un constructo complejo, amplio, multifactorial y requiere de abordajes multiactor y multinivel, no obstante, y a pesar de lo previamente mencionado, no se debe dejar de lado los aportes que las EAAA, como las mencionadas en el presente caso de estudio, constituyen un paso hacia adelante, hacia la construcción de un modelo de abasto alimentario sostenible, de producción y consumo local.

El estudio de caso desarrollado en este texto permite observar que, a pesar de las posibles debilidades mostradas por este tipo de estructura de abasto alimentario, el caso presentado es ampliamente resiliente. Sin embargo, aun cuando este tipo de estructura de CCC muestra gran capacidad adaptativa, es importante mencionar que, para su sostenibilidad, requieren de una serie de entramados institucionales fortalecidos que le permitan solventar, no solamente las adversidades comunes y vicisitudes continuas en el quehacer alimentario, sino atravesar contextos adversos de gran magnitud, como lo acontecido en la CDMX y la emergencia sanitaria. 


\section{BIBLIOGRAFÍA}

Azevedo, C. (2009). La configuración de los circuitos "de proximidad" en el sistema alimentario: tendencias evolutivas. Documents d'anàlisi geogràfica, (54). https://core.ac.uk/download/pdf/13285626.pdf

Bassols, Á., Torres, F., Delgadillo, J., Chías, L., Gasca, J., Peña, E. y Echánove, F. (1994). El abasto alimentario en las regiones de México. Universidad Nacional Autónoma de México, Instituto de Investigaciones Económicas y Programa Universitario de Alimentos.

Chiffoleau, Y., Millet-Amrani, S., Rossi, A., Rivera-Ferre, M. G. y López Merino, P. (2019). The participatory construction of new economic models in short food supply chains. Journal of Rural Studies, 68. https://doi. org/10.1016/j.jrurstud.2019.01.019

Delgadillo, M. y Sanz, J. (2018). Sistemas agroalimentarios locales de proximidad: contextos rururbanos en México y España. Universidad Nacional Autónoma de México, Coordinación de Humanidades, Instituto de Investigaciones Económicas.

Dowler, E. A., Kneafsey, M., Lambie, H., Inman, A. y Collier, R. (2011). Thinking about "food security": engaging with UK consumers. En Y. Chiffoleau, S. Millet-Amrani, A. Rossi, M. G. Rivera-Ferre y P. López Merin. The participatory construction of new economic models in short food supply chains. Journal of Rural Studies, 68. https://doi.org/10.1016/j. jrurstud.2019.01.019

Goodman, D., DuPuis, E. M. y Goodman, M. K. (2012). Alternative food networks: knowledge, practice, and politics. Ed. Routledge Taylor \& Francis Group.

Instituto Interamericano de Cooperación para la Agricultura (IICA) (2020). Monitor para la seguridad alimentaria de las Américas. https://www.iica. int/es/monitor?utm_medium=Email\&utm_source=Comunicado $+\mathrm{de}+\mathrm{pr}$ ensa $\% 3 \mathrm{~A}+\mathrm{El}+\mathrm{IICA}+\mathrm{lanza}+\mathrm{un}+$ Monitor+con+informaci\%C3\%B3n+clav e+sobre+seguridad+alimentaria+en+Am\%C3\%A9rica+Latina+y+el+Cari be\&\#

Korhonen, K., Kotavaara, O., Muilu, T. y Rusanen, J. (2017). Accessibility of local food production to regional markets-case of Berry production in Northern Ostrobothnia, Finland. European Countryside, 9(4). https://doi. org/10.1515/euco-2017-0040

López, A. y Agencia El Universal (2011). Mercado “El 100”, opción orgánica de jóvenes emprendedores. https://vanguardia.com.mx/mercadoel100opcionorganicadejovenesemprendedores-642536.html 
Marsden, T. y Morley, A. (2014). Sustainable food systems: Building a new paradigm. En L. Reina-Usuga, T. De-Haro y C. Parra-López. Canales cortos de comercialización territoriales: conceptualización, caracterización y aplicación para el caso de Córdoba. ITEA Información Técnica Económica Agraria. Revista de la Asociación Interprofesional para el Desarrollo Agrario AIDA, 114(2). https://doi.org/10.12706/itea.2018.012

Mercado El 100 (M100) (2020). Mercado el 100. Primer mercado de productores locales, orgánicos y ecológicos en la CDMx. https://mercadoel100. org/tag/presentamos/

Organización de las Naciones Unidas para la Agricultura y la Alimentación (fAO) (1996). Declaración de Roma sobre la Seguridad Alimentaria Mundial, Cumbre Mundial sobre la Alimentación. http://www.fao.org/3/ w3613s/w3613s00.htm\#: - :text=Reafirmamos\%20que\%20un\%20entorno $\% 20$ pol\%C3\%ADtico,la\%20erradicaci $\% C 3 \% B 3$ n $\% 20$ de $\% 20$ la $\% 20$ pobreza.

(2016). Circuitos Cortos de Comercialización: una mirada desde el enfoque territorial. http://www.fao.org/inaction/territoriosinteligentes/ articulos/colaboraciones/detalle/s/c/410218/

Organización de las Naciones Unidas para la Agricultura y la Alimentación y Unión Europea (FAO y UE) (2010). ¿Qué es la seguridad alimentaria? Conceptos y marcos de Seguridad Alimentaria para la Acción. http://www.fao. org/elearning/course/FC/es/pdf/trainerresources/learnernotes0531.pdf

Padilla, R. y Oddone, N. (2016). Manual para el fortalecimiento de cadenas de valor. Sede Subregional de la Comisión Económica para América Latina y el Caribe (CEPAL), No. 40662. https://repositorio.cepal.org/bitstream/ handle/11362/40662/1/S1601085_es.pdf

Pardo, J. y Durand, L. (2018). Los mercados alternativos de alimentos en la Ciudad de México. Medio ambiente, sustentabilidad y vulnerabilidad social. En J. J. Cervantes Niño, L. Márquez Mireles y D. Molina Rosales (coords.). Las ciencias sociales y la agenda nacional. Reflexiones y propuestas desde las ciencias sociales. vol. 5 Medio Ambiente, sustentabilidad y vulnerabilidad social (pp. 470-488). comecso. https://www.comecso.com/cienciassociales-agenda-nacional/cs/article/view/1273/458

Pensado, M. del R. (2003). La evolución del consumo de frijol en la Ciudad de México durante la década de los 90. En G. Torres Salcido (coord.). Politicas de abasto alimentario alternativas para el Distrito Federal y su Zona Metropolitana (pp. 197-222). FCPys-UnAM.

Reina-Usuga, L., De-Haro, T. y Parra-López, C. (2018). Canales cortos de comercialización territoriales: conceptualización, caracterización y aplica- 
ción para el caso de Córdoba. ITEA Información Técnica Económica Agraria. Revista de la Asociación Interprofesional para el Desarrollo Agrario AIDA, 114(2). https://doi.org/10.12706/itea.2018.012

Renting, H., Marsden, T. K. y Banks, J. (2003). Understanding alternative food networks: Exploring the role of short food supply chains in rural development. Environment and Planning A: Economy and Space, 35(3). https://doi.org/10.1068/a3510

Salgado-Sánchez, R. y Castro-Ramírez, A. E. (2016). Mercado el 100, experiencia de consumo participativo para favorecer la sustentabilidad de la agricultura y los sistemas alimentarios. Agricultura, Sociedad y Desarrollo, 13(1). http://www.scielo.org.mx/scielo.php?script=sci_arttext\&pid $=$ S1870-54722016000100105

Secretaría de Desarrollo Social (sedesol) (2016). Diagnóstico de la Seguridad Alimentaria y Nutricional de la Ciudad de México. http://data.evalua. cdmx.gob.mx/docs/estudios/edf2016/e_DiagSegAlim_DF.pdf

Time Out México (2020). Mercado El 100: una alternativa ecológica para consumir local. https://www.timeoutmexico.mx/ciudad-de-mexico/restaurantes/mercado-el-100

Urquía, N. (2014). La seguridad alimentaria en México. Salud Pública de México, 56(1). http://www.scielo.org.mx/pdf/spm/v56s1/v56s1a14.pdf

Yin, R. (2003). Case study research. Design and methods. SAGE Editors. https:// www.saludpublica.mx/index.php/spm/issue/view/293 